\title{
A CULTURA DO COCO: UMA ANÁLISE ECONÔMICA ${ }^{1}$
}

\author{
MÔNICA DE MOURA PIRES², ROBERTA SANTOS COSTA³, ABEL REBOUÇAS SÃO JOSÉ4, MOEMA MARIA BADARÓ \\ CARTIBANI MIDLEJ ${ }^{5}$, JAÊNES MIRANDA ALVES ${ }^{6}$
}

\begin{abstract}
RESUMO - O coqueiro é uma planta de clima tropical, sendo cultivado em cerca de 90 países, destacando-se o continente asiático na produção e comercialização do produto in natura e nos subprodutos. Podem-se aproveitar diversas partes da planta, como o fruto, as folhas, a inflorescência, entre outros produtos e subprodutos. O Brasil possui condições especiais que favorecem a adaptação e o desenvolvimento da cultura do coco. Dentre as principais regiões brasileiras produtoras, o Nordeste destaca-se, produzindo cerca de $80 \%$ de toda a produção nacional. A Bahia destacase como um dos principais produtores e consumidores. O município de Ilhéus vem expandindo a produção ao longo do tempo, em função do fluxo de turistas e de iniciativas de diversificar a agricultura regional. O objetivo deste estudo é identificar e avaliar, econômica e financeiramente, os diferentes níveis tecnológicos adotados pelos produtores de coco no município de Ilhéus. Neste estudo, utilizou-se de levantamento de corte seccional (crosssection) para o qual foram aplicados vinte questionários junto aos produtores, selecionados por meio do critério de amostra intencional, segundo a técnica survey. Por meio dessas informações, foram calculados os indicadores financeiros (VPL, TIR, B/C) e a análise de sensibilidade. Nota-se que os três níveis tecnológicos adotados no município apresentam receitas positivas; no entanto, a tecnologia III é a que se mostra mais eficiente. Os resultados, para as tecnologias I, II e III, do VPL são de R \$ 4.857,04; R \$20.782,38, e R \$ 47.931,22, respectivamente; para a TIR, de 43\%, 80\% e 87\%, sucessivamente. A relação B/C foi de 2,32 (Tecnologia I), 3,24 (Tecnologia II) e 2,89 (Tecnologia III). Tais resultados indicam que as tecnologias adotadas são econômica e financeiramente viáveis. No entanto, o produtor poderia realizar maior exploração do potencial da atividade, gerando maior lucratividade.
\end{abstract}

Termos para indexação: Cocus nucifera, tecnologia de produção, viabilidade financeira.

\section{COCONUT CULTIVATION: AN ECONOMICAL ANALYSIS}

ABSTRACT - Coconut is a tropical tree being cultivated in about 90 countries, standing out the Asian continent in production and commercialization of the product "in natura" and in processed products. It is possible to take advantage of several parts of the tree, such as fruit, leaves, inflorescence, among others. Brazil presents good environmental conditions for adaptation and development of coconut cultivation. Among the main brazilian production areas, the Northeast stands out as the main producer (about $80 \%$ of national production). Bahia State stands out as one great producer and consumer. Ilhéus town in Bahia State is expanding the production along the time, in function of the tourists' flow and initiatives of diversification of the regional agriculture. The objective of this study was identifying and evaluating, economical and financially, the different technological levels adopted by the producers of coconut in Ilhéus Region. In this study it was used the survey cross-section technique for which twenty questionnaires were applied to the producers, selected through the criterion of intentional sample, according to the survey technique. Through those information the financial indicators were calculated (VPL, TIR, B/C) and sensibility analysis. It is noticed that the three technological levels adopted in the studied region presented positive results, however the Technology III is the most efficient. The results, for the Technologies I, II and III, of positive VPL are of $\mathrm{R} \$ 4,857.04, \mathrm{R} \$ 20,782.38$ and $\mathrm{R} \$ 47,931.22$, respectively; for TIR of $43 \%, 80 \%$ and $87 \%$, successively. The relationship B/C was of 2.32 (Technology I), 3.24 (Technology II) and 2.89 (Technology III). Such results indicate that the adopted technologies are economical and financially viable. However, the producer can increase technology use to get higher advantages of the activity in order of generating larger profitability.

Index Terms: Cocus nucifera, production technology, financial viability.

Atualmente, o Brasil possui cerca de 50 mil hectares cultivados com coqueiro-anão, distribuídos, praticamente, em quase todo o território nacional. As maiores plantações encontram-se na faixa litorânea do Nordeste, com uma produção, no ano de 2001, superior a 960 mil frutos, ou seja, aproximadamente, $68 \%$ de toda a produção nacional. Em nível estadual, a Bahia destaca-se, pois sua produção atingiu cerca de $30 \%$ do total nacional e $44 \%$ da produção nordestina em 2001 (424.444 frutos), sendo o maior produtor. Em seguida, têm-se os estados do Ceará e de Sergipe com 203.769 frutos e 90.413 frutos, respectivamente (IBGE, 2003).

Dentre as regiões baianas produtoras de coco, o Sul do Estado possui condições edafoclimáticas favoráveis, além de constituir-se em importante região consumidora, principalmente nos meses de maior fluxo de turista (dezembro a fevereiro). Dentre os municípios que fazem parte dessa região, destacam-se Camamu, Cairu, Canavieiras, Una e Ilhéus (IBGE, 2003). A produção dessa região, em especial do município de Ilhéus, tem apresentado tendência de crescimento, porém com algumas oscilações nos últimos anos.

Em função da rentabilidade financeira e do crescente consumo da água de coco nos grandes centros urbanos, tem havido interesse de produtores pela cultura. Aliado aos aspectos positivos do mercado, o coqueiro, dependendo da tecnologia utilizada, pode florescer com, aproximadamente, dois anos de idade e atingir, em função dos tratos culturais dispensados, mais de 200/frutos/pé/ano, o que proporciona maior rapidez dos investimentos realizados. Como o coco é um produto agrícola de boa aceitação no mercado quando comparado a outras culturas agrícolas, pode-se adotá-lo como uma alternativa de diversificação da agricultura da região sudeste baiana, em especial do município de Ilhéus (Mororó, 1998; Tonet, 2000; Aragão, 2001). Tais argumentos embasam o estudo econômico dessa atividade e da análise de viabilidade frente aos diferentes níveis tecnológicos empregados na produção de coco, no município de

\footnotetext{
${ }^{1}$ (Trabalho 041/2003). Recebido: 25/03/2003. Aceito para publicação: 14/01/2004.

${ }^{2}$ DS em Economia Rural, Universidade Estadual de Santa Cruz, Departamento de Ciências Econômicas, Rod. Ilhéus-Itabuna, km 16 - Salobrinho, CEP 45650-000 Ilhéus-BA, tel.: (73)680-5215-e-mail: mpires@uesc.br.

${ }^{3}$ Bacharel em Ciências Econômicas, Universidade Estadual de Santa Cruz, Departamento de Ciências Econômicas, Rod. Ilhéus-Itabuna, km 16 - Salobrinho, CEP 45650-000 Ilhéus-BA, tel.: (73)680-5215.

${ }^{4}$ DS em Fitotecnia, Universidade Estadual do Sudoeste da Bahia, Departamento de Fitotecnia e Zootecnia, Estrada do Bem Querer, km 4, CEP 45100-000 - Vitória da Conquista-BA, tel.: (77)424-8600 - e-mail: abelsj@uesb.br.

${ }^{5}$ MS em Economia Rural, Universidade Estadual de Santa Cruz, Departamento de Ciências Econômicas, Rod. Ilhéus-Itabuna, km 16 - Salobrinho, CEP 45650-000 Ilhéus-BA, tel.: (73)680-5215 - e-mail: midlej@uol.com.br.

${ }^{6}$ DS em Economia Aplicada, Universidade Estadual de Santa Cruz, Departamento de Ciências Exatas e Tecnológicas, Rod. Ilhéus-Itabuna, km 16 - Salobrinho, CEP 45650-000 Ilhéus-BA, tel.: (73)680-5200 - e-mail: jaenes@uesc.br.
} 
Ilhéus.

O objetivo geral deste estudo foi identificar as tecnologias empregadas pelos produtores de coco de Ilhéus, analisando a viabilidade econômico-financeira da cultura para o município.

A abrangência do presente estudo refere-se ao município de Ilhéus, em função dos aspectos edafoclimáticos favoráveis para a cultura do coco, e por constituir-se em pólo turístico do Estado da Bahia. Esses aspectos tornam-se relevantes na produção e comercialização do coco.

Neste estudo, utilizou-se de levantamento de corte seccional (cross-section) para o qual foram entrevistados vinte produtores, selecionados por meio do critério de amostra intencional, segundo a técnica survey, por meio de informações dos cadastros da CEPLAC. As visitas aos produtores foram realizadas no período de setembro de 2001 a janeiro de 2002, nas fazendas da região Sapucaeira, onde se concentram $80 \%$ das propriedades com produção de coco. A partir desses questionários, determinaram-se os fatores de produção e os níveis de receita. Os preços dessas variáveis foram determinados para março de 2002. Os custos de produção foram elaborados considerando desde a implantação, formação e produção comercial (a partir do $3^{\circ}$ ano), para um horizonte de planejamento de 20 anos.

A partir das informações obtidas nas entrevistas, foram elaborados três intervalos de produtividade, em função do uso de insumos (defensivos e fertilizantes). O primeiro intervalo, denominado de Tecnologia I, relaciona a produtividade de 1 a 50 frutos/pé/ano; o segundo, de Tecnologia II, com produtividade variando de 51 a 100 frutos/pé/ano, e o terceiro, de Tecnologia III, com produtividade entre 101 e 150 frutos/pé/ano. Essas diferenças de produtividade estão relacionadas aos tratos culturais dispensados à cultura, especialmente no que diz respeito ao uso de fertilizantes e defensivos. Os produtores classificados na Tecnologia I realizam consórcio e não conseguem utilizar as doses recomendadas de fertilizantes e defensivos, em função da sua limitação de recurso. Os produtores da Tecnologia III são aqueles que utilizam as doses recomendadas, além de realizarem adequadamente os tratos culturais, tendo o coco como atividade principal. Quanto aos produtores da Tecnologia II, apesar de procurarem adequar-se ao recomendado para a cultura, possuem limitações de gasto.

Para a análise econômico-financeira da produção de coco, utilizaram-se três instrumentos de avaliação: valor presente líquido (VPL), taxa interna de retorno (TIR) e relação benefício/custo (B/C), conforme Contador (1988). O valor presente líquido (VPL), refere-se ao benefício líquido do projeto, atualizando a determinada taxa de desconto. A taxa interna de retorno (TIR) é o valor da taxa de desconto que torna o valor presente líquido igual a zero, e a relação benefício/ custo (B/C) é a relação entre os benefícios e os custos atualizados. Matematicamente,

$$
\begin{aligned}
& V P L=\sum_{i=0}^{n}(R i-C i) /(1+t)^{i} \\
& \sum_{i=0}^{n}(R i-C i) /\left(1+t^{*}\right)^{i}=0 \\
& B / C=\sum_{i=0}^{n}\left[(R i) /(1+t)^{i}\right] / \sum_{i=0}^{n}(C i) /(1+t)^{i}
\end{aligned}
$$

em que, $\mathrm{R}_{\mathrm{i}}$ corresponde aos benefícios da atividade no ano $\mathrm{i} ; \mathrm{C}_{\mathrm{i}}$ corresponde aos custos da atividade no ano i, inclusive investimentos; $t$ é a taxa de desconto relevante ao período i; $t^{*}$ é a taxa interna de retorno relevante ao período $\mathrm{i}$.

Neste estudo, adotou-se como taxa de atratividade o rendimento acumulado da caderneta de poupança de 12 meses, de $8,49 \%$ (novembro de 2000 a outubro de 2001), para os três níveis tecnológicos. Foram feitas simulações no preço da mão-de-obra e no preço do esterco para os três níveis tecnológicos, adotando-se aumentos de 10 e $20 \%$ no preço dessas variáveis.

Como na região estudada há uma predominância da cultura de coco-anão, a pesquisa limitou-se apenas às informações referentes a essa variedade.

Na região Sapucaeira, há uma diversificação agrícola relativamente grande. Em geral, os produtores utilizam as áreas agricultáveis com várias culturas, como mandioca, cacau, banana, cupuaçu, piaçava, café, mangustão, graviola, maracujá, bem como hortaliças e leguminosas. Mesmo assim, para cerca de $75 \%$ dos agricultores entrevistados, a cultura do coco representa sua atividade principal. Sendo que $20 \%$ dos coqueirais do município encontram-se em formação e $80 \%$ estão em fase de produção. A maior parte das propriedades está concentrada em áreas inferiores a 100 hectares (65\%), sendo que $35 \%$ dos produtores estão classificados em áreas superiores a 100 hectares. Essas diferenças no tamanho das propriedades refletem no nível tecnológico empregado pelo produtor e, conseqüentemente, na produtividade. Para os pequenos produtores de coco, a mandioca e os seus subprodutos representam a maior fonte de renda. Para os médios e grandes produtores, a cocoicultura representa a atividade agrícola que possibilita maiores ganhos de renda.

Em geral, os produtores têm sido orientados pela CEPLAC, para adotarem espaçamento de 7,5m x 7,5m x 7,5m, com 205 mudas por hectare. No entanto, verificaram-se alguns plantios com espaçamento diferente, em função de que os produtores associavam o coco a outras culturas, como forma de minimizar os riscos financeiros.

O destino do coco produzido no município de Ilhéus é, basicamente, o mercado local. Dependendo do destino, os canais de comercialização possuem seis fluxos básicos. Em geral, os produtores vendem diretamente o produto in natura para os intermediários, os atacadistas, os varejistas ou até mesmo ao consumidor final. Quando o fruto é industrializado, os produtores deslocam a produção para a indústria local ou intermediários das indústrias. Assim, para o produto in natura atingir o consumidor final, pode-se ter até três elos anteriores no fluxo de comércio. Para o produto industrializado, têmse, em geral, quatro canais anteriores para atingir o consumidor final.

Quando há excedentes, a produção segue para outros Estados, como São Paulo, Rio de Janeiro e Espírito Santo. Esses mercados, porém, são mais exigentes com relação aos aspectos de qualidade do fruto (sabor e aspectos físicos) e uniformidade no tamanho, o que, muitas vezes, restringe a comercialização do produto naqueles mercados.

Atualmente, o mercado comprador considera o tamanho do fruto o fator mais importante na definição do preço pago ao produtor. Além dessas exigências específicas do processo produtivo e de colheita, existe a concorrência entre os pequenos e os grandes produtores. Esses últimos, em geral, em função do volume produzido, conseguem atingir maior número de mercados, concorrendo via preço. Como tem havido interesse de produtores locais na industrialização da água de coco, a agroindústria processadora desse produto poderá representar o grande mercado para o fruto. Salienta-se, porém, que a maioria das agroindústrias encontra-se fora do Estado da Bahia, estando localizadas nos Estados de Sergipe, Alagoas, Pernambuco, Paraíba e Rio Grande do Norte, o que aumenta os custos em função do transporte para essas localidades.

As maiores despesas com a atividade foram observadas com o uso da Tecnologia III; no entanto, o nível de produtividade dos coqueirais compensa os custos, pois as condições técnicas adotadas proporcionam um nível de produtividade duas vezes superior à produtividade da Tecnologia II (média de 67 frutos/pé/ano). Este valor aumenta, consideravelmente, quando comparado à Tecnologia I, que é, em média, 24 frutos/pé/ano (4.920 frutos por hectare) (Costa, 2002).

No entanto, observou-se que as diferenças tecnológicas não influenciavam no início da produção, que ocorria a partir do terceiro ano; porém, nos anos subsequientes, a utilização não adequada de insumos refletia nos níveis de produtividade, tornando-os 
TABELA 1 - Despesa, receita e fluxo de caixa para os três níveis tecnológicos, para 1 hectare de coco, no município de Ilhéus, em 2001

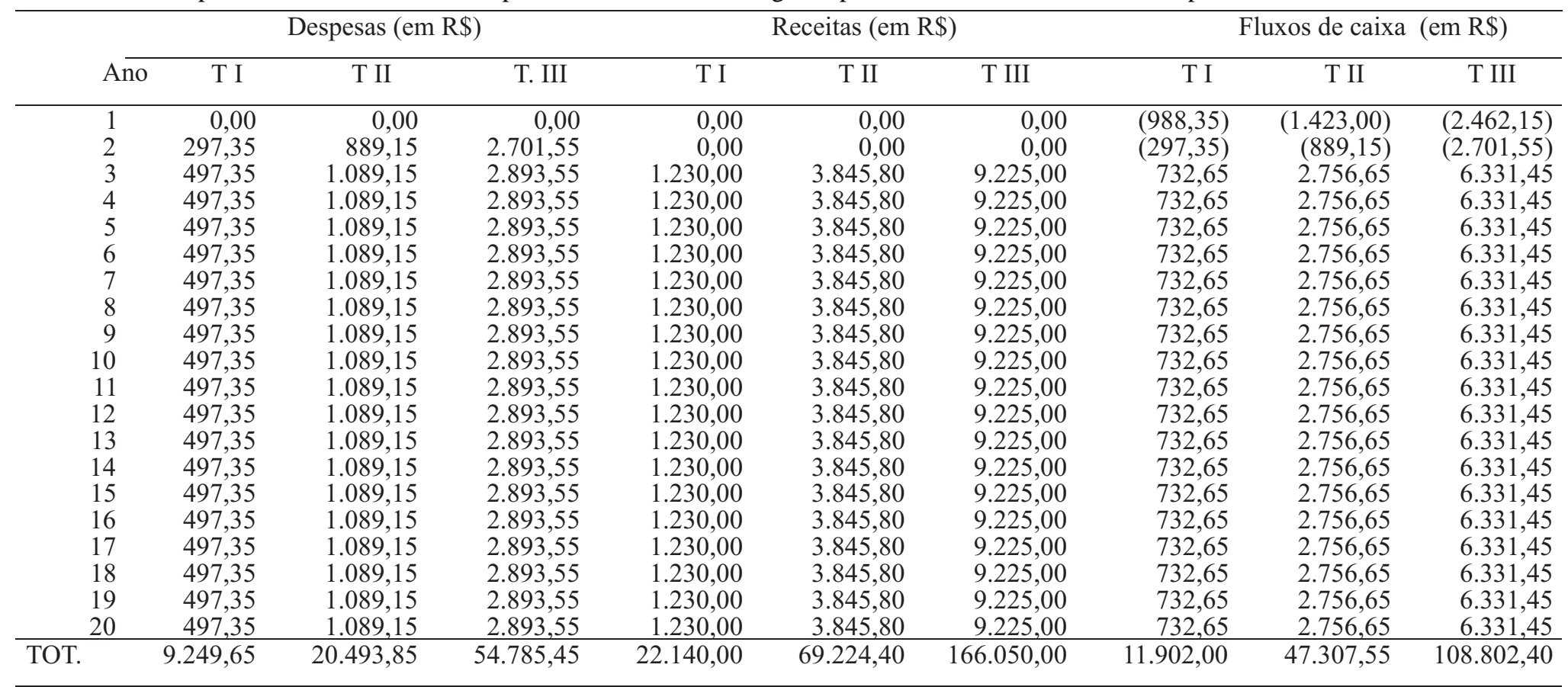

T I - Tecnologia I, T II - Tecnologia II e T III - Tecnologia III.

Fonte: Dados da pesquisa.

TABELA 2 - Custo de produção, receita total e lucro total nos três níveis tecnológicos, para 1 hectare de coco, no município de Ilhéus, em 20 anos

\begin{tabular}{lcrrr}
\hline $\begin{array}{l}\text { Níveis } \\
\text { Tecnológicos }\end{array}$ & \multicolumn{2}{c}{ Custos de produção(em R\$) } & $\begin{array}{c}\text { Receita Total } \\
\text { (em R\$) }\end{array}$ & $\begin{array}{c}\text { Lucro Bruto } \\
(\text { em R } \$)\end{array}$ \\
\cline { 2 - 4 } Tecnologia I & Custos Fixos & Custos Variáveis & $22.140,00$ \\
Tecnologia II & - & $9.249,65$ & $12.890,35$ \\
Tecnologia III & - & $20.493,35$ & $69.224,40$ \\
\end{tabular}

Fonte: Dados da pesquisa.

consideravelmente menores quando comparados a tecnologias mais intensivas no uso de insumos e no manejo mais adequado da cultura.

Os níveis de preço do produto foram $\mathrm{R} \$ 0,25 /$ fruto (Tecnologia I), R\$0,28/fruto (Tecnologia II) e R \$0,30/fruto (Tecnologia III). Essa diferença de preço está relacionada às características físicas do fruto (coloração e tamanho), sabor da água e outros aspectos associados à qualidade.

Para a Tecnologia I, o investimento foi de R\$998,35, com uma receita média anual de $\mathrm{R} \$ 1.230,00$. Para a Tecnologia II, as receitas foram maiores em função do preço de venda ter sido $11,2 \%$ superior ao preço do produto comercializado pelos produtores classificados na Tecnologia I. Na Tecnologia III, o nível de investimento com a cultura é maior, isso interferiu no total das despesas dos produtores. Porém, como a produtividade e o preço recebido por fruto são maiores, estes conseguem obter melhores resultados financeiros com a atividade, comparativamente aos produtores que adotaram as Tecnologias I e II. Dessa forma, os maiores investimentos com a atividade e o preço recebido pelo produtor definiram as diferenças tecnológicas e, conseqüentemente, as receitas e as despesas dos produtores (Tabela 1).

Para as Tecnologias I e II, os custos fixos foram relativamente inexpressivos, adotando-os como nulos. Na Tecnologia III, a utilização de maquinários proporcionou custo fixo anual de $\mathrm{R} \$ 2.400,00$. Os custos variáveis, no final de vinte anos, para a Tecnologia I, foram de $\mathrm{R} \$ 9.249,65$, de $\mathrm{R} \$ 20.493,85$ para a Tecnologia II, e $\mathrm{R} \$ 54.785,45$ para a Tecnologia III. As receitas totais foram de R $\$ 22.140,00 ; \mathrm{R} \$ 69.224,40$, e R\$166.050,00, para os níveis I, II e III, respectivamente. Tais resultados propiciaram lucro bruto de $\mathrm{R} \$ 12.890,35$ para o nível I; R 48.730,55 para o nível II, e 108.864,55 para o nível III (Tabela 2).
Esses resultados configuram um potencial da atividade para a região em estudo. No entanto, deve-se ressaltar que outros elementos externos, especificamente, as medidas de política governamental, em nível municipal, estadual e/ou federal, afetam a expectativa de ganho com a atividade. Assim, o produtor deve estar atento às condições de mercado, realizando acompanhamento do seu empreendimento, bem como dos seus concorrentes.

Conforme a Tabela 3, todas as tecnologias apresentaram VPL positivo e TIR superior à taxa de desconto de $8,49 \%$ (poupança). No entanto, os melhores resultados foram obtidos com a Tecnologia III. Os valores encontrados para a relação benefício/custo foram superiores à unidade, ou seja, benefícios esperados maiores que os custos. O maior valor foi obtido com a Tecnologia II $(3,24)$, o que significa que, para cada unidade monetária investida na atividade, deve-se obter um retorno de $\mathrm{R} \$ 2,24$. Esses resultados indicam que as tecnologias adotadas pelos produtores do município de Ilhéus apresentam viabilidade financeira; assim, a cocoicultura pode constituir-se em uma alternativa viável de investimento e diversificação agrícola. As simulações propostas neste trabalho não afetaram a viabilidade da atividade (Tabela 4 ).

TABELA 3 - Valor presente líquido (VPL), Taxa interna de retorno (TIR), relação benefício/custo $(\mathrm{B} / \mathrm{C})$ dos três níveis tecnológico, para 1 hectare de coco, no município de Ilhéus, em 2001

\begin{tabular}{llll}
\hline Níveis Tecnológicos & VPL & TIR & B/C \\
\hline Tecnologia I & $4.857,04$ & $43 \%$ & 2,32 \\
Tecnologia II & $20.782,38$ & $80 \%$ & 3,24 \\
Tecnologia III & $47.931,22$ & $87 \%$ & 2,89 \\
\hline
\end{tabular}

Fonte: Dados da pesquisa. 
TABELA 4 - Simulação de oscilações no preço da mão-de-obra e no preço do esterco, para a implantação de 1 hectare de coco, no município de Ilhéus, em 2001

\begin{tabular}{|c|c|c|c|c|c|c|c|c|c|}
\hline \multirow{3}{*}{$(\%)$} & \multicolumn{8}{|c|}{ Mão-de-obra } & \\
\hline & \multicolumn{3}{|l|}{ VPL } & \multicolumn{3}{|l|}{ TIR } & \multicolumn{3}{|l|}{$\mathrm{B} / \mathrm{C}$} \\
\hline & T 1 & T II & T III & T 1 & T II & T III & T 1 & T II & T III \\
\hline 0 & $4.857,04$ & $20.782,38$ & $47.931,22$ & $43 \%$ & $80 \%$ & $87 \%$ & 2,32 & 3,24 & 2,89 \\
\hline 10 & $4.672,76$ & $20.538,69$ & $47.632,00$ & $41 \%$ & $78 \%$ & $85 \%$ & 2,25 & 3,17 & 2,86 \\
\hline \multirow[t]{4}{*}{20} & $4.488,48$ & $20.295,00$ & $47.322,79$ & $39 \%$ & $76 \%$ & $84 \%$ & 2,18 & 3,11 & 2,84 \\
\hline & \multicolumn{9}{|c|}{ Esterco } \\
\hline & \multicolumn{3}{|l|}{ VPL } & \multicolumn{3}{|l|}{ TIR } & \multicolumn{3}{|l|}{$\mathrm{B} / \mathrm{C}$} \\
\hline & T 1 & T II & T III & T 1 & T II & T III & $\mathrm{T} 1$ & T II & T III \\
\hline 0 & $4.857,04$ & $20.782,38$ & $47.931,22$ & $42,8 \%$ & $80 \%$ & $87 \%$ & 2,32 & 3,24 & 2,89 \\
\hline 10 & $4.840,08$ & $19.056,75$ & $47.417,50$ & $42,6 \%$ & $75 \%$ & $85 \%$ & 2,31 & 3,02 & 2,84 \\
\hline 20 & $4.823,13$ & $20.295,00$ & $46.903,79$ & $42,0 \%$ & $68 \%$ & $83 \%$ & 2,30 & 2,79 & 2,79 \\
\hline
\end{tabular}

T I - Tecnologia I, T II - Tecnologia II e T III - Tecnologia III.

Fonte: Dados da pesquisa.

As diferenças tecnológicas identificadas estão diretamente relacionadas às condições físicas da cultura e do aporte financeiro do produtor, que se traduzem nos tratos culturais dispensados à cultura, tendo impacto positivo sobre os indicadores (VPL, TIR, B/C) e, conseqüentemente, na lucratividade do empreendimento.

A Tecnologia III apresentou os melhores resultados financeiros para o produtor de coco comparativamente aos outros níveis tecnológicos analisados. Mesmo assim, verifica-se que as tecnologias alternativas empregadas no município proporcionam resultados positivos, conforme os indicadores analisados. Esses indicadores, porém, mostraram-se poucos sensíveis a alterações nos preços dos insumos (mão-de-obra e esterco).

Tais resultados indicam que as tecnologias adotadas são econômica e financeiramente viáveis. No entanto, a lucratividade poderia ser maior caso o produtor empregasse mais adequadamente os fatores de produção, pois melhores frutos proporcionam maior nível de preço, o que poderia ampliar as receitas com a atividade.

Nesse contexto, uma política capaz de potencializar o mercado de coco na Bahia, especificamente no município de Ilhéus, deve preocupar-se com aumentos de produção e eficiência no processo de comercialização do produto, vislumbrando a possibilidade de agregação de valor ao produto, como a instalação de agroindústrias na região.

\section{REFERÊNCIASBIBLIOGRÁFICAS}

ARAGÃO, W.M. A importância do coqueiro-anão verde. Disponível em: <http://www.embrapa.br>. Acesso em: 25 out. 2001.

CONTADOR, C. R. Avaliação social de projetos. 2. ed. São Paulo: Atlas, 1988.316p.

COSTA, R. S. Análise econômica da cultura do coco no município de Ilhéus, Bahia, 2002. 61f. Monografia (Trabalho de Graduação em Ciências Econômicas) - Universidade Estadual de Santa Cruz, 2002. IBGE - INSTITUTO BRASILEIRO DE GEOGRAFIA E ESTATÍSTICA. Disponível em: <http://www.ibge.gov.br>. Acesso em: 18 ago. 2003.

MORORÓ, R. C. Industrialização do coco: manual sobre a industrialização dos derivados do coco, Ilhéus: CEPLAC/CPT CPT, 1998.60p.

PIRES, M. de M.; SÃO JOSÉ, A. R.; BRAGA, M. J.; RUFINO, J. L. dos. Aspectos econômicos da cultura de coco no Brasil. In: SÃO JOSÉ, A. R. et al. COCO: produção e mercado. Vitória da Conquista: DFZ/UESB, 1999. p. 7-19.

TONET, R. M.. Cultura do coqueiro: uma tomada de decisão técnica e de mercado. Disponível em: <http://www.cati.sp.gov.br/notícias/ m_pesquisaartigo.html >. Acesso em: maio 2000. 